\title{
Design Theory
}

DOI 10.1007/s12599-010-0128-2

\section{The Authors}

\author{
Christian Fischer MSc. IS \\ Prof. Dr. Robert Winter ( $\varangle)$ \\ Dr. Felix Wortmann \\ Institute of Information Management \\ University of St. Gallen \\ Müller-Friedberg-Strasse 8 \\ 9000 St. Gallen \\ Switzerland \\ Christian.Fischer@unisg.ch \\ Robert.Winter@unisg.ch \\ Felix.Wortmann@unisg.ch
}

Received: 2010-08-24

Accepted: 2010-09-08

Accepted after one revision by

Prof. Dr. Sinz.

Published online: 2010-10-20

This article is also available in German in print and via http://www. wirtschaftsinformatik.de: Fischer C, Winter R, Wortmann F (2010) Gestaltungstheorie. WIRTSCHAFTSINFORMATIK. doi: 10.1007/s11576-0100245-z.

(c) Gabler Verlag 2010

\section{Motivation}

Design Science Research for Information Systems (ISDSR) has received considerable attention recently. With the growing interest in ISDSR, calls continue to establish the rigor of artifact construction. In analogy to other scientific disciplines, the scientific foundation of artifact construction has been designated as IS Design Theory (ISDT) (Gregor 2006, p. 611). Although the ISDSR community has been discussing ISDTs since the early 1990s, no consensus on the definition or the componential structure of ISDTs has been reached yet. In this short article, we give an overview of the ongoing discussion on ISDTs. First, we introduce fundamental concepts of ISDT. Second, we give an overview on seminal contributions to the field of ISDTs in chronological order. Finally, we cluster the presented ISDT contributions into ISDT schools.

\section{Design Theory Fundamentals}

\subsection{Theory in Social Sciences}

In general, the purpose of a theory is explanation and prediction (Walls et al. 1992 , p. 38). While this statement is true for natural as well as for social sciences, these disciplines have a different approach towards theory (Walls et al. 1992, p. 40). Goals as objects of study are key for social sciences - but they are meaningless in natural sciences (Walls et al. 1992, p. 40). Even when focusing on social sciences, different views on theory do exist (Gregor 2006, p. 616). Abstraction and generalization about phenomena, interactions, and causations are recognized as the core of every theory (Gregor 2006, p. 616). We therefore elaborate on these elementary concepts in the following.

Building upon Chmielewicz, two different kinds of statements can be distinguished (Chmielewicz 1994, p. 10). On the one hand, singular statements (e.g., single artifact instances) do not generalize or abstract from particular facts. On the other hand, general statements (e.g., reference models) do abstract from particular facts and serve as a basis to derive singular statements that solve practical problems. Definitions and terms describing phenomena are the building blocks for both classes of statements. General statements can be differentiated into three types (Chmielewicz 1994, pp. 8 10):

- Theoretical statements describe causeeffect relationships, e.g., "higher utilization leads to lower unit costs". Explanation and prediction are key goals of theoretical statements.

- Technological statements express means-end relationships, e.g., "for reducing unit costs, increasing utilization is an effective measure". By setting effects as goals (ends) and causes as means, theoretical statements can be transformed into means-end relationships.

- Theoretical and technological statements represent facts. There is no judgment if the implementation of a technological statement is a desired action. In contrast, normative statements are based on a value or goal system. They specify concrete choices on the basis of goal priority and known side effects, e.g., unit costs should be reduced by improving employee productivity and not by decreasing employee salary. Value statements are often made implicitly, e.g., profit orientation is assumed, but not explicitly mentioned as a fundamental value assumption.

Building upon the three classes of general statements, we discuss theory in the context of ISDSR in the following section.

\subsection{Theory in ISDSR}

ISDSR "seeks to extend the boundaries of human and organizational capabilities by creating new and innovative artifacts" (Hevner et al. 2004, p. 75) instead of analyzing given phenomena. Artifacts are built and evaluated in a design process (Hevner et al. 2004, pp. 79-80) and can be of various types, e.g., methods or models (March and Smith 1995). Theories can play a dual role in a design process (Venable 2006a, p. 185): On the one hand, artifact construction can be grounded on theory. On the other hand, theory can be the outcome of a design process ("IS design theory", ISDT). While the use of theories as a basis for artifact construction is well accepted, the question whether a theory can be the outcome of a design process is part of the ongoing scientific discourse.

In order to facilitate the interpretation of ISDT literature, we briefly characterize key theory types:

- Explanatory theory: An explanatory theory is a set of theoretical statements that provides explanations (Gregor 2006, p. 620).

- Predictive theory: A predictive theory is a set of theoretical statements that provides predictions. A predictive theory can also be an explanatory theory and vice versa (Gregor 2006, p. 620).

- Normative theory: A normative theory is a set of normative statements.

- Prescriptive theory: A prescriptive theory integrates explanatory and predictive theory as well as normative statements for artifact construction (Gregor 2006, p. 620; Walls et al. 1992, p. 41). 
While this classification clearly distinguishes theoretical statements and normative statements, it does not explicitly define technological statements, but "blends" them into prescriptive statements.

Building upon this classification, ISDT literature distinguishes kernel and design theories. There is a broad consensus that kernel theories are theories from natural or social sciences and serve as a foundation for artifact construction (Walls et al. 1992, p. 42). It remains however unclear if kernel theories have to fulfill additional criteria, e.g., being explanatory and/or predictive.

Goal orientation and means-end relationships are at the core of design theories (Walls et al. 1992, p. 40). Therefore, technological statements form the basis of an ISDT. It is discussed controversially whether an ISDT contains normative and/or prescriptive statements. Moreover, there is a debate on the relationship between artifact and ISDT: What essential criteria distinguish an "ordinary" artifact from an ISDT? Finally, the precise relationship between kernel theories and ISDTs remains vague in ISDT literature. Two controversial positions exist: (1) Kernel theories are defined as fundamental theories being used throughout the design process for construction. Following this definition, an ISDT can be a kernel theory. (2) Kernel theories are defined as explanatory and/or predictive theories from natural or social sciences. On the basis of this definition, ISDTs and kernel theories are mutually exclusive.

\section{Design Theory in IS Literature}

In the following, we present six influential ISDT contributions in chronological order. Our presentation focuses on answering three key questions that are discussed controversially in literature: (1) Some authors refer to the term artifact, others use the term design theory. Is there a need for an explicit ISDT? (2) Some authors say that a theoretical grounding is mandatory for an ISDT, others say a theoretical grounding is nice to have but unnecessary. Thus, we ask: Are kernel theories required to ground ISDTs? (3) Finally, some authors say that an instance is a valid ISDSR output, others claim for generality. Hence, we ask: What is the required degree of generality of ISDSR outputs i.e., is a single instance a valid ISDSR output? Most of the articles to be discussed in the following have been published in A/B journals. Our selection and summary of relevant literature are inspired by Venable's (2006b) excellent overview.

Walls et al. (1992) are the first to describe characteristics and components of an ISDT. They define an ISDT as "a prescriptive theory based on theoretical underpinnings which says how a design process can be carried out in a way which is both effective and feasible" (p. 37). ISDTs differ from other theory types in IS as they specify how to reach a given goal. In addition to an enumeration of seven characteristics of ISDTs (pp. 40-41), they define components of an ISDT (p. 42). For them, the components of a design product are (1) a class of requirements ("meta-requirements"), (2) a class of artifacts hypothesized to meet the "metarequirements" ("meta-design"), (3) theories from natural or social sciences governing design requirements ("kernel theories"), and (4) testable design product hypotheses used to test whether the "meta-design" satisfies the "metarequirements"; those of a design process are (1) a design method describing procedures for artifact construction, (2) kernel theories, and (3) testable design process hypotheses (Walls et al. 1992, Table 4). Kernel Theories: For Walls et al., it is essential that design theories are grounded on explanatory, predictive, or normative theories and show how such theories can be put to practical use (Walls et al. 1992, p. 41). Generality: Walls et al. claim that a design theory states how to reach a class of goals (Walls et al. 1992, p. 42), not a single goal. They do not consider single instances to be a design theory.

March and Smith (1995) do not explicitly write about design theories, but about ISDSR in general. They differentiate between descriptive "natural science" ${ }^{1}$ and prescriptive "design science" (for description vs. prescription cf. pp. 252, 254). "Whereas natural science tries to understand reality, design science attempts to create things that serve human purposes" (p. 253). Consequently, they argue that theory building is characteristic for natural science and not part of design science. Kernel Theories: March and Smith do not make any statement about whether or not to use kernel theories for artifact construction (cf. also Kuechler and Vaishnavi (2008a, 2008b, Table 1). Generality: March and Smith explicitly say that an "instance" is a valid ISDSR output. For them, generality is not a characteristic of ISDSR outputs.

Hevner et al. (2004) oppose behavioral sciences to design science. Like March and Smith (1995), they do not use the term "design theory". ${ }^{2}$ Their main contribution consists of a set of seven guidelines for ISDSR. Kernel Theories: Hevner et al. mention the IS knowledge base that is comprised of results of behavioral sciences as well as design science research. If helpful, such knowledge may be used for constructing design science artifacts - but ISDSR artifacts do not necessarily need to be grounded on kernel theories. Generality: Hevner et al. take the attitude of March and Smith (1995) that instances are a valid output of ISDSR. Although not clearly expressed, Hevner et al. seem to presuppose that an artifact should satisfy a certain degree of generality.

Goldkuhl (2004) reflects the grounding of ISDTs. In his opinion, the use of ISDTs informs the design process (p. 61). $\mathrm{He}$ differentiates between four types of grounding (Goldkuhl 2004, Fig. 3): (1) Conceptual grounding deals with the appropriate use of constructs of a design theory. (2) By value grounding, Goldkuhl means a justification of the goals and values of a design theory, including those of underlying (sub-)rationalities. (3) Explanatory grounding means that explanatory theories are used for justifying design theories or elements of it. (4) Empirical grounding refers to an empirical evaluation of an ISDT e.g., in a case study. Kernel Theories: Concerning kernel theories, Goldkuhl writes: "As opposed to Walls et al. (1992) I do not conceive kernel theories (explanatory theories) to be indispensible parts of design theories" (p. 66). Generality: Goldkuhl does not explicitly address the generality of a design theory. Nevertheless, with respect to ISDTs, he argues that "not all practical knowledge should be considered to be design theories. Only theorized practical knowledge should be conceived as design theory" (p. 61).

\footnotetext{
${ }^{1}$ By "natural science", March and Smith do not only mean the traditional natural sciences, such as physics, chemistry, or biology, but research in all fields, including social and behavioral sciences (p. 253), which aims at describing, explaining, and/or predicting phenomena.

${ }^{2}$ It should be noted that Salvatore March is co-author both of March and Smith (1995) and Hevner et al. (2004).
} 
Building upon the earlier work by Walls et al. (1992), Gregor and Jones (2007) criticize the componential ISDT structure for its "unnecessary complexity" (p. 315). They propose a simplified structure with six core components (1-6) and two additional components $(7,8)$ : (1) Purpose and scope, (2) constructs, (3) principles of form and function, (4) artifact mutability, (5) testable propositions, (6) justificatory knowledge, (7) principles of implementation, and (8) expository instantiation (Gregor and Jones 2007, Table 2). Kernel Theories: Although seeing difficulties concerning the use of kernel theories, Gregor and Jones argue "that it remains essential to include justificatory knowledge in ISDTs, although this knowledge could be incomplete" (p. 328). They therefore consider justificatory knowledge i.e., kernel theories, to be a core component of ISDTs. Generality: Gregor and Jones write that requirements defined in the purpose and scope of an ISDT "are not the requirements for one instance of a system" (p. 325). Consequently, they argue "that 'constructs, models and methods' are all one type of thing and can be equated to theory or components of theory, while instantiations are a different type of thing altogether" (p. 320).

Venable (2006b) sees ISDTs as a special kind of "utility theory" linking a technology X (from a solution space) to a problem Y (from a problem space), thereby predicting a certain amount of utility. An ISDT therefore needs only three components: technology, problem, and utility. Furthermore, in contrast to all other authors described above, he argues that "the idea that a design theory should be prescriptive [...] does not seem appropriate" (Venable 2006b, p. 12). In general, multiple means-end relationships can be leveraged in order to reach a certain goal. Rather than applying one prescriptive theory, sophisticated decision making is necessary. Kernel Theories: Venable pragmatically argues that kernel theories are nice to have, but should not be considered as a necessity. "Explanations of how and why solutions work, while important, aren't the core issue. The fact [...] that they do work and how well is the issue" (Venable 2006b, p. 11). Generality: Venable (2006b) clearly says that "Design Science Research [... ] should be related to a generalized (or abstracted), type, kind, or class of problems that are relevant to typical, identified classes of stakeholders" (p. 10).

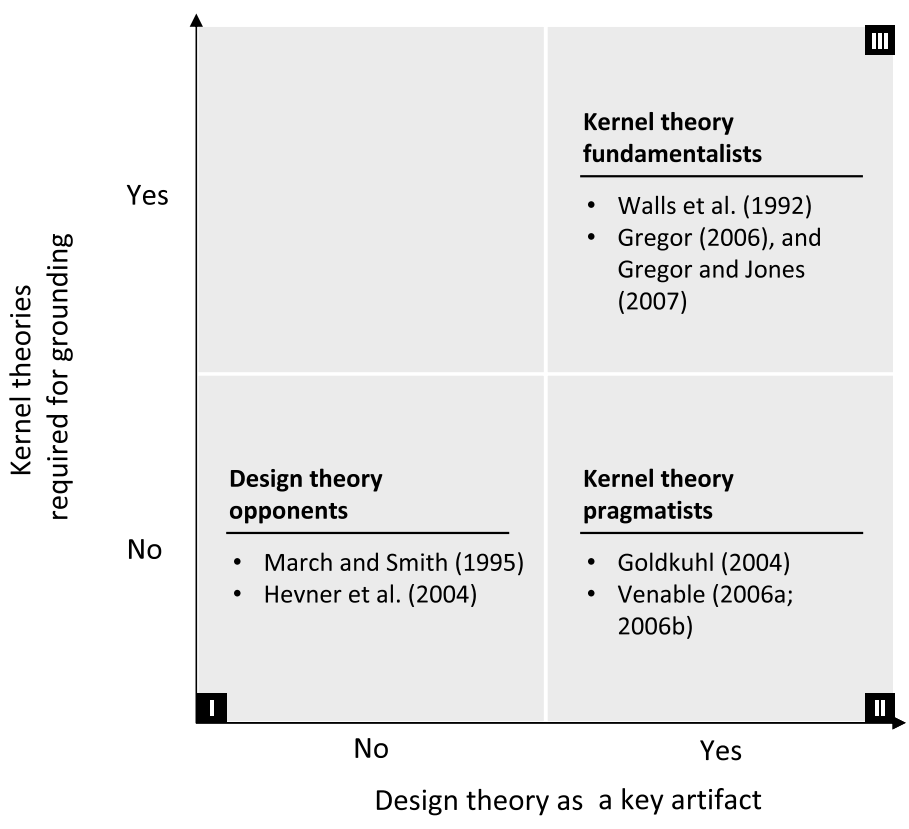

Fig. 1 Design theory "schools"

\section{Conclusion}

Theory in social sciences is based on theoretical, technological as well as normative statements. Key to ISDTs are technological statements which link means to goals. In order to identify similar attitudes towards ISDTs, we classify the presented ISDT contributions with regard to two dimensions:

(1) "Design theory as a key artifact" depicts whether or not ISDTs are recognized as essential outputs of ISDSR. The respective authors' attitude towards ISDTs directly relates to the attitude towards a mandatory degree of artifact generality and vice versa. Seeing a strong need for abstraction and generalization in ISDSR motivates to create a separate type of "high quality" artifacts in respect to these criteria (i.e., ISDT artifacts).

(2) "Kernel theories required for grounding" reflects whether or not kernel theories are considered to be mandatory for artifact construction.

Figure 1 illustrates the classification and allows to distinguish three different "ISDT schools":

(I) Design theory opponents: Both March and Smith (1995) and Hevner et al. (2004) do not consider ISDTs as key outputs for ISDSR. Furthermore, they do not emphasize the importance of kernel theories for artifact construction.
(II) Kernel theory pragmatists: Goldkuhl (2004) and Venable (2006b) see the need for ISDTs, but do not insist on a kernel theory-based grounding of ISDTs. Goldkuhl emphasizes the importance of grounding, but considers kernel theories as only one way of grounding. Venable points out the importance of artifact impact over artifact grounding.

(III) Kernel theory fundamentalists: Walls et al. (1992), Gregor (2006), and Gregor and Jones (2007) claim that ISDTs are an output of ISDSR. In addition, they call for kernel theories as mandatory components of ISDTs.

\section{References}

Chmielewicz K (1994) Forschungskonzeptionen der Wirtschaftswissenschaft. Schäffer-Poeschel, Stuttgart

Goldkuhl G (2004) Design theories in information systems - a need for multi-grounding. Journal of Information Technology Theory and Application 6(2):59-72

Gregor S (2006) The nature of theory in information systems. MIS Quarterly 30(3):611642

Gregor S, Jones D (2007) The anatomy of a design theory. Journal of the Association for Information Systems 8(5):312-335

Hevner AR, March ST, Park J, Ram S (2004) Design science in information systems research. MIS Quarterly 28(1):75-105

Kuechler B, Vaishnavi VK (2008a) On theory development in design science research - anatomy of research project. European Journal of Information Systems 17(5):489504 
Kuechler B, Vaishnavi VK (2008b) Theory development in design science research anatomy of a research project. In: Vaishnavi VK, Baskerville RL (eds) Third international conference on design science research in information systems and technology, pp 115

March ST, Smith GF (1995) Design and natural science research on information technol- ogy. Decision Support Systems 15(4):251266

Venable J (2006a) A framework for design science research activities. In: Emerging trends and challenges in information technology management. Idea Group, Hershey, pp 184-187

Venable J (2006b) The role of theory and theorising in design science research. In: Chat- terjee $S$, Hevner AR (eds) First international conference on design science in information systems and technology. Claremont Graduate University, Claremont, pp 1-18

Walls JG, Widmeyer GR, El Sawy OA (1992) Building an information system design theory for vigilant EIS. Information Systems Research 3(1):36-59 